\title{
BMJ Open Biological and clinical correlates of the patient health questionnaire-9: exploratory cross-sectional analyses of the baseline health study
}

\author{
Robert M Califf (D , ${ }^{1}$ Celeste Wong, ${ }^{1}$ P Murali Doraiswamy, ${ }^{2,3}$ David S Hong, ${ }^{4}$ \\ David P Miller, ${ }^{1}$ Jessica L Mega, ${ }^{1}$ Baseline Study Group
}

To cite: Califf RM, Wong C, Doraiswamy PM, et al. Biological and clinical correlates of the patient health questionnaire-9: exploratory cross-sectional analyses of the baseline health study. BMJ Open 2022;12:e054741. doi:10.1136/ bmjopen-2021-054741

- Prepublication history and additional supplemental material for this paper are available online. To view these files, please visit the journal online (http://dx.doi.org/10.1136/ bmjopen-2021-054741).

Received 23 June 2021 Accepted 08 December 2021

Check for updates

(c) Author(s) (or their employer(s)) 2022. Re-use permitted under CC BY-NC. No commercial re-use. See rights and permissions. Published by BMJ.

${ }^{1}$ Verily Life Sciences LLC, South San Francisco, California, USA ${ }^{2}$ Department of Psychiatry and Behavioral Sciences, Duke University School of Medicine, Durham, North Carolina, USA

${ }^{3}$ Duke Institute for Brain

Sciences, Duke University

School of Medicine, Durham,

North Carolina, USA

${ }^{4}$ Department of Psychiatry and Behavioral Sciences, Stanford University School of Medicine, Stanford, California, USA

Correspondence to

Dr Robert M Califf;

robert.califf@duke.edu

\section{ABSTRACT}

Objectives We assessed the relationship between the Patient Health Questionnaire-9 (PHQ-9) at intake and other measurements intended to assess biological factors, markers of disease and health status.

Design, setting and participants We performed a crosssectional analysis of 2365 participants from the Baseline Health Study, a prospective cohort of adults selected to represent major demographic groups in the USA. Participants underwent deep phenotyping on demographic, clinical, laboratory, functional and imaging findings. Importance Despite extensive research on the clinical implications of the PHQ-9, data are limited on the relationship between $\mathrm{PHQ}-9$ scores and other measures of health and disease; we sought to better understand this relationship.

Interventions None.

Main outcomes and measures Cross-sectional measures of medical illnesses, gait, balance strength, activities of daily living, imaging and laboratory tests. Results Compared with lower PHQ-9 scores, higher scores were associated with female sex $(46.9 \%-66.7 \%)$, younger participants (53.6-42.4 years) and compromised physical status (higher resting heart rates (65 vs $75 \mathrm{bpm})$, larger body mass index $\left(26.5-30 \mathrm{~kg} / \mathrm{m}^{2}\right)$, greater waist circumference $(91-96.5 \mathrm{~cm}))$ and chronic conditions, including gastro-0esophageal reflux disease $(13.2 \%-24.7 \%)$ and asthma $(9.5 \%-20.4 \%)(p<0.0001)$. Increasing PHQ-9 score was associated with a higher frequency of comorbidities (migraines $(6 \%-20.4 \%)$ ) and active symptoms (leg cramps $(6.4 \%-24.7 \%)$, mood change $(1.2 \%-47.3 \%)$, lack of energy $(1.2 \%-57 \%)$ ) $(p<0.0001)$. After adjustment for relevant demographic, socioeconomic, behavioural and medical characteristics, we found that memory change, tension, shortness of breath and indicators of musculoskeletal symptoms (backache and neck pain) are related to higher $\mathrm{PHQ}-9$ scores $(p<0.0001)$.

Conclusions Our study highlights how: (1) even subthreshold depressive symptoms (measured by PHQ-9) may be indicative of several individual- and populationlevel concerns that demand more attention; and (2) depression should be considered a comorbidity in common disease.

Trial registration number NCT03154346.
Strengths and limitations of this study

- This study included deep phenotyping on demographic, clinical, laboratory, functional and imaging findings of 2365 participants from the Baseline Health Study.

- This study provides important data on the relationship between Patient Health Questionnaire- 9 scores and other measures of health and disease.

- The cross-sectional nature of this study limits our ability to assess the time course of these findings; however, follow-up is currently accruing.

- People with significant depression are less likely to volunteer, thereby limiting the breadth of depression observed in this study.

- The study population is generally representative of adult age, sex, race and ethnicity, but it is not a fully representative sample of the population.

\section{INTRODUCTION}

Depression is a complex, chronic condition that affects hundreds of millions of people worldwide. ${ }^{1}$ Bidirectional relationships have been reported between depression and many chronic illnesses ${ }^{2}$; however, most studies have focused on specific conditions, such as diabetes, stroke or congestive heart failure, as opposed to a multidimensional deep phenotyping approach. Findings from previous studies highlight the need to more fully characterise the relationship between depression and physical health. ${ }^{3-5}$ Furthermore, it would be helpful to better understand whether these relationships exist only above a certain threshold or across the entire continuum.

The most common screening tool for depression is the Patient Health Questionnaire-9 (PHQ-9), whose operating characteristics are well known ${ }^{6}$ and have been validated in a variety of contexts. ${ }^{78}$ Despite extensive research on the clinical and behavioural implications of PHQ-9, ${ }^{9} 10$ the results of this 
questionnaire are often used in a dichotomous manner (eg, cut-off of 10) without evaluating the full relationship between PHQ-9 scores and measures of health and disease.

The Baseline Health Study (BHS) ${ }^{11}$ is a prospective cohort study of an adult population selected to represent major demographic groups in the USA. In BHS, deep phenotyping of numerous demographic, clinical, laboratory, functional and imaging findings is coupled with ongoing longitudinal follow-up. The purpose of our study was to assess the relationship between the entire spectrum of depression, measured by PHQ-9, and a broad array of measurements intended to assess health status.

\section{METHODS}

\section{The Baseline Health Study}

BHS methods have been previously described, ${ }^{11-13}$ including entry and exclusion criteria, the institutional review board and participant consent procedures, the data collection scheme and key components of study procedures.

Additional details of the effects of social determinants on health in the BHS study have been previously reported. ${ }^{12}$ BHS is enrolling a large number of participants, beginning with intensive measurement of the first 2502 people (the deeply phenotyped cohort) in whom a large volume of multimodal data are collected. Four clinical BHS sites in the USA have begun enrolment.

The participants were enrolled through a virtual online registry with selection of participants for the deep phenotyping cohort included in this report using an algorithm intended to produce a representative sample of USA adult age, race and ethnicity. People in good health and with medical conditions were included and the sampling method was designed to over-represent people at risk of heart disease or cancer. The PHQ-9 in this report was collected at the initial study visit in person or online.

A pre-BHS pilot study, which tested clinical assessment workflows, was conducted in 200 healthy participants prior to initiation of the primary study. BHS is funded by Verily (South San Francisco, California, USA) and is managed in collaboration with Stanford University (Stanford, California, USA), Duke University (Durham, North Carolina, USA, and the California Health and Longevity Institute (Westlake Village, California, USA) with enrolling sites in Durham, North Carolina; Kannapolis, North Carolina; Los Angeles, California and Palo Alto, California, USA. The extended studies have governance approaches specific to the needs of each study. Herein, we examine a cross-sectional analysis of the first BHS time point PHQ-9 scores.

\section{Statistical methods}

The statistical methods used in this manuscript have been described previously. ${ }^{12}$ Distributional measures; medians and 25th, 75th percentiles for continuous variables; and counts and percentages for categorical variables were computed and summarised across each of 5 PHQ-9 severity groups ${ }^{6}(0,1-4,5-9,10-14,>14)$, divided by convention to be consistent with prior studies. The Cochran-Armitage trend test for binomial variables, ${ }^{13} 14$ and the Spearman rank correlation test for continuous variables $^{15}$ or categorical variables that are ordinal in nature (eg, education and income), were used to test for linear trend across severity group. Multiple tests were not adjusted for, given the exploratory nature of this study. Subsequent studies with preplanned hypotheses are needed to confirm results.

Penalised regression using the least absolute shrinkage and selection operator (LASSO) was conducted to model physical, phenotypic and symptom factors that could predict the PHQ-9 score (logarithm of PHQ-9 +1). Data were randomly split into a training set (approximately $70 \%$ of the data), which was used to build the models, and an independent test set, which was used to evaluate model performance. The final linear model was trained on the full training set, retaining all predictors with coefficients not equal to zero, and was evaluated on the heldaside test set.

Since inference rather than prediction is the goal of this analysis, we considered five sets of sequential 'adjustment' models, consisting of smaller-to-larger covariate lists that were entered into a LASSO regression model. The LASSO-predicted values resulting from each of the five models were used to estimate a covariate-adjusted effect for all other candidate variables. Separate regressions for each of the candidate variables that are not included in a given model were performed to obtain coefficients and CIs (ie, only the LASSO-predicted value and the candidate variable to be evaluated were included in each model).

The LASSO adjustment models comprise the following covariates: (1) model 1: age, sex, and age $\times$ sex interaction; (2) model 2: variables from model 1, plus race and ethnicity, socioeconomic-related variables (highest education completed, household income, marital status, employment status and health insurance) and behavioral-related variables (smoking status, pack-years smoked, and the Alcohol Use Disorders Identification Test-Consumption sum score); (3) model 3: variables from model 2 plus medical conditions except mental health disorder diagnoses or disorders directly related to mental health or depression (major depressive disorder, generalised anxiety disorder, attention deficit hyperactivity disorder, post-traumatic stress disorder, bipolar disorder, alcohol abuse, drug abuse and concussion or loss of consciousness); (4) model 4: variables from model 3 , plus symptoms and allergies, except those symptoms that are directly related to mental health or depression (ie, nervousness, mood changes, fatigue, lack of energy, change in sleep patterns, change in appetite and difficulty concentrating) and (5) model 5: variables from model 4, plus all physical health metrics. Patient-reported outcomes, including measures of anxiety, psychological and social well-being (eg, General Anxiety Disorder-7, 
WHO Disability Assessment Schedule, and Satisfaction with Life), and self-reported medical conditions and symptoms related to mental health or depression, have been reported ${ }^{6}$ and were excluded from these analyses to enable a focus on physical and medical findings. In preliminary analyses, higher PHQ-9 score was associated with female sex, age, and the age by sex interaction, confirming the well-established relationship between depression, sex and age, ${ }^{16}{ }^{17}$ thereby informing our decision to include age and sex in all models. The key variables included in each covariate model can be found in online supplemental eTable 1.

Since LASSO regression techniques require an input dataset with complete data, missing data were addressed using iterative regression-based imputation, where predictors were first grouped by data type.Groups were rankordered by the most missing to the least missing data. The rank of the whole group was based on the amount of missingness of the majority $(\geq 50 \%)$ of the fields within that group), and then at each imputation step, the grouped predictors were used in a regression model to predict the missing data. The PHQ-9 score was imputed in the last step along with other patient-reported outcome scores and, therefore, all 2502 participants were included in the LASSO models.

To summarise key model findings adjusted for known demographic and socioeconomic-related risk factors of PHQ-9, 4 groups were created based on the LASSOpredicted value using model 1 (age, sex, and age $\times$ sex interaction) and model 2 (race, ethnicity, socioeconomicrelated variables and health behaviours). For both models, 'high risk' was defined as the top 50\% of the predicted value, while 'low risk' was defined as the bottom $50 \%$ of the predicted value. The two risk groups from each model were combined to create the following four groups: (1) high-risk model 1+high-risk model 2; (2) high-risk model 1+low-risk model 2; (3) low-risk model 1+high-risk model 2; and (4) low-risk mdel 1+low risk model 2.

\section{Patient and public involvement statement}

From the beginning, BHS has used participant and community engagement methods. Participants have been involved in feedback sessions, and return of results is a commitment of the project. Community meetings have also been held to assure broad feedback on the goals and conduct of the project.

\section{RESULTS}

The relationship between the PHQ-9 score and key demographic characteristics and vital signs is shown in table 1 . Younger participants, women, people of colour and those of Hispanic ethnicity had higher PHQ-9 scores. While no difference was observed in blood pressure or temperature, higher PHQ-9 scores were found in participants with higher resting heart rates, larger body mass index, greater waist circumference and higher respiratory rates.
PHQ-9 scores as a function of medical history and symptoms are shown in table 2 and online supplemental eTable 2. As expected, participants with other chronic conditions, particularly gastro-oesophageal reflux disease, anxiety and asthma, had higher PHQ-9 scores. A history of diagnosed depression was highly correlated with elevated PHQ-9. Various symptoms were evident in participants with higher PHQ-9 scores, with musculoskeletal, mood and anxiety symptoms proving particularly prominent.

Table 3 demonstrates the relationship between measures of physical performance and PHQ-9; differences in $6 \mathrm{~min}$ walk distance, handgrip strength, leg balance, chair stand and mean steps are particularly associated with higher PHQ-9.

The covariates resulting from each LASSO model are shown in online supplemental eTables 3-7. All other variables that are not presented in those tables are set to zero by the LASSO regression and thereby excluded from the final adjustment models. Age remained a top predictor in all models. Where health behaviours and measures of socioeconomic status were included (ie, models 2-5), smoking status and employment, insurance and marital status also remained in the models as significant predictors. When medical conditions were added (ie, models 3-5), respiratory-related conditions such as asthma remained in the models, and when symptoms were added (ie, models 4 and 5), tension, body image concerns and memory change remained in the models as top predictors. Model performance was similar between the training and test sets for each model (model $1: \mathrm{R}^{2}=0.032$ vs 0.035 ; model 2: $\mathrm{R}^{2}=0.117$ vs 0.112 ; model 3: $\mathrm{R}^{2}=0.167$ vs 0.133 ; model 4: $\mathrm{R}^{2}=0.247$ vs 0.228 ; and model $5: \mathrm{R}^{2}=0.270$ vs 0.240 , respectively).

The interplay of higher PHQ-9 score predictors based on the LASSO regression adjustment models is shown in figures 1-5. Regardless of adjustment factors included in the models, memory change, tension, shortness of breath and indicators of musculoskeletal symptoms are significantly related to higher PHQ-9 scores (models 1-3). Further details of the LASSO analysis are included in online supplemental eTables 8-12. Tension, memory change, and back pain consistently remain in the models after adjustment. When medical conditions, symptoms, and allergies are taken into account, indicators of obesity (body mass index, waist circumference) and lack of physical fitness are the most significant predictors of PHQ-9 score. When physical performance is also factored into the adjustment, laboratory values are only weakly associated with PHQ-9 scores. online supplemental eFigures 1-5 present the distribution of PHQ-9 according to a sample of other health conditions, such as asthma, sleep apneoa and body image concerns, after adjustment for known demographic and socioeconomic-related risk factors of PHQ-9. The inter-relationships are clear across this spectrum of measures, emphasising the importance of understanding all three dimensions when designing interventions. 
Table 1 Demographics: PHQ-9 score

\begin{tabular}{|c|c|c|c|c|c|}
\hline & PHQ-9 0 (N=484) & $\begin{array}{l}\text { PHQ-9 1-4 } \\
(\mathrm{N}=1086)\end{array}$ & PHQ-9 5-9 (N=518) & $\begin{array}{l}\text { PHQ-9 10-14 } \\
(\mathrm{N}=184)\end{array}$ & PHQ-9 15+ (N=93) \\
\hline $\begin{array}{l}\text { Age, median (25th, } \\
\text { 75th) }\end{array}$ & $53.6(36.7,66.1)$ & $51.7(37.0,66.5)$ & $47.6(32.7,60.7)$ & $42.8(31.2,55.1)$ & $42.4(32.1,54.2)$ \\
\hline Female sex ${ }^{*}$ & $227(46.9)$ & $622(57.3)$ & $299(57.7)$ & $108(58.7)$ & $62(66.7)$ \\
\hline \multicolumn{6}{|l|}{ Race } \\
\hline Black & $80(16.5)$ & $153(14.1)$ & $82(15.8)$ & $31(16.8)$ & $19(20.4)$ \\
\hline Asian† & $64(13.2)$ & $113(10.4)$ & $47(9.1)$ & $15(8.2)$ & $5(5.4)$ \\
\hline $\mathrm{NHOPI}$ & $7(1.4)$ & $11(1)$ & $7(1.4)$ & $2(1.1)$ & $0(0.0)$ \\
\hline $\begin{array}{l}\text { American Indian or } \\
\text { Alaska Native }\end{array}$ & $4(0.8)$ & $8(0.7)$ & $11(2.1)$ & $3(1.6)$ & $1(1.1)$ \\
\hline Other† & $35(7.2)$ & $70(6.4)$ & $48(9.3)$ & $23(12.5)$ & $11(11.8)$ \\
\hline Los Angeles & $94(19.4)$ & $194(17.9)$ & $111(21.4)$ & 35 (19) & $21(22.6)$ \\
\hline Durham & $99(20.5)$ & $196(18.0)$ & $102(19.7)$ & $40(21.7)$ & $25(26.9)$ \\
\hline Kannapolis & $100(20.7)$ & $226(20.8)$ & $105(20.3)$ & 41 (22.3) & $28(30.1)$ \\
\hline Palo Alto† & $191(39.5)$ & $470(43.3)$ & $200(38.6)$ & $68(37.0)$ & $19(20.4)$ \\
\hline $\begin{array}{l}\text { Systolic BP, median } \\
\text { (25th, 75th), mm Hg }\end{array}$ & $123.0(112.5,133.1)$ & $122.0(112.0,132.5)$ & $123.0(112.5,133.5)$ & $119.5(109.9,132.0)$ & $122.5(115.5,130.5)$ \\
\hline $\begin{array}{l}\text { Diastolic BP, median } \\
\text { (25th, 75th), mm Hg }\end{array}$ & $75.5(69.0,82.0)$ & $75.0(68.0,82.0)$ & $76.0(70.0,83.5)$ & $74.0(68.9,81.1)$ & $77.5(71.5,84.0)$ \\
\hline $\begin{array}{l}\text { Body mass index, } \\
\text { median (25th, } 75 \text { th), kg/ } \\
\mathrm{m}^{2 \star}\end{array}$ & $26.5(23.7,29.9)$ & $26.1(22.9,31.0)$ & $28.0(24.5,33.2)$ & $29.1(25.1,34.7)$ & $30.0(25.4,38.8)$ \\
\hline $\begin{array}{l}\text { Oxygen saturation, } \\
\text { median ( } 25 \text { th, } 75 \text { th), \%† }\end{array}$ & $99.0(98.0,100.0)$ & $99.0(98.0,100.0)$ & $99.0(98.0,100.0)$ & $99.0(97.0,100.0)$ & $98.0(97.0,100.0)$ \\
\hline
\end{tabular}

Data shown are no (\%), unless otherwise indicated.

$\mathrm{P}$ values for trend were calculated with the use of Spearman correlation or Cochrane-Armitage tests, where appropriate.

${ }^{*} P$ value for trend $<0.0001$.

$\dagger \mathrm{P}$ value for trend $<0.01$.

BP, blood pressure; NHOPI, Native Hawaiians and other Pacific Islanders; PHQ-9, Patient Health Questionnaire-9.

\section{DISCUSSION}

Our study reinforces previous observations regarding the relationship between PHQ-9 and measures of chronic disease and poor physical performance in addition to the critical importance of social factors and psychological distress, previously published. ${ }^{12}$ While these findings are not surprising, they highlight how PHQ-9 is an entree into a variety of individual-level and population-level concerns that demand more attention. Contextual awareness is critically important when the PHQ-9 is used in clinical practice ${ }^{18}$ as recommended by the USA Preventive
Services Task Force ${ }^{19}$ for screening within a health system or for public health assessment.

This analysis cannot answer questions of cause and effect since it is cross-sectional. The PHQ-9 and detailed serial measures of biological, clinical, behavioural and social function will be assessed in the ongoing BHS longitudinal study; the bi-directional relationship between the PHQ-9 and these multiple measures will be particularly interesting, as few studies have collected this amount of detail in a diverse population. This measurement depth of demographic, clinical, biological and behavioural issues 
Table 2 Medical history: PHQ-9 score

\begin{tabular}{|c|c|c|c|c|c|}
\hline & $\begin{array}{l}\text { PHQ-9 } 0 \\
(\mathrm{~N}=484)\end{array}$ & $\begin{array}{l}\text { PHQ-9 1-4 } \\
(\mathrm{N}=1086)\end{array}$ & $\begin{array}{l}\text { PHQ-9 5-9 } \\
(\mathrm{N}=518)\end{array}$ & $\begin{array}{l}\text { PHQ-9 10-14 } \\
(\mathrm{N}=184)\end{array}$ & $\begin{array}{l}\text { PHQ-9 15+ } \\
(\mathrm{N}=93)\end{array}$ \\
\hline Alcohol use disorder* & $1(0.2)$ & $20(1.8)$ & $12(2.3)$ & $7(3.8)$ & $6(6.5)$ \\
\hline Fibromyalgia* & $2(0.4)$ & $10(0.9)$ & $11(2.1)$ & $7(3.8)$ & $6(6.5)$ \\
\hline Bipolar disorder* & $3(0.6)$ & $9(0.8)$ & $12(2.3)$ & $9(4.9)$ & 8 (8.6) \\
\hline PTSD* & $8(1.7)$ & $14(1.3)$ & $15(2.9)$ & $21(11.4)$ & $9(9.7)$ \\
\hline COPD with emphysema* & $5(1.0)$ & $19(1.7)$ & $22(4.2)$ & $12(6.5)$ & $10(10.8)$ \\
\hline $\mathrm{ADHD}^{*}$ & $15(3.1)$ & $50(4.6)$ & $33(6.4)$ & $25(13.6)$ & $11(11.8)$ \\
\hline Asthma* & $46(9.5)$ & $157(14.5)$ & $79(15.3)$ & $39(21.2)$ & $19(20.4)$ \\
\hline Migraine $^{\star}$ & $29(6.0)$ & $134(12.3)$ & 77 (14.9) & $31(16.8)$ & $19(20.4)$ \\
\hline GERD* & $64(13.2)$ & $176(16.2)$ & $106(20.5)$ & $40(21.7)$ & $23(24.7)$ \\
\hline Anxiety* & $13(2.7)$ & $98(9.0)$ & $109(21.0)$ & 55 (29.9) & $34(36.6)$ \\
\hline Depression* & $16(3.3)$ & $93(8.6)$ & $110(21.2)$ & $63(34.2)$ & $55(59.1)$ \\
\hline Itching skin* & $19(3.9)$ & $81(7.5)$ & $50(9.7)$ & 30 (16.3) & $10(10.8)$ \\
\hline Sinus pain* & $18(3.7)$ & $77(7.1)$ & $58(11.2)$ & $21(11.4)$ & $10(10.8)$ \\
\hline Urgency ${ }^{\star}$ & $13(2.7)$ & $69(6.4)$ & $46(8.9)$ & $23(12.5)$ & $10(10.8)$ \\
\hline Excessive belching or passing of gas ${ }^{\star}$ & $24(5.0)$ & $84(7.7)$ & $54(10.4)$ & $29(15.8)$ & $13(14.0)$ \\
\hline Dryness* $^{*}$ & $35(7.2)$ & $113(10.4)$ & $68(13.1)$ & $39(21.2)$ & $14(15.1)$ \\
\hline Heartburn* & $27(5.6)$ & $94(8.7)$ & $70(13.5)$ & $42(22.8)$ & $15(16.1)$ \\
\hline Dry mouth* & $27(5.6)$ & $84(7.7)$ & $64(12.4)$ & $28(15.2)$ & $16(17.2)$ \\
\hline Constipation* & $22(4.5)$ & $98(9.0)$ & $64(12.4)$ & $48(26.1)$ & $17(18.3)$ \\
\hline Numbness or loss of sensation* & $17(3.5)$ & $54(5.0)$ & $38(7.3)$ & $19(10.3)$ & $17(18.3)$ \\
\hline Cramping* & $14(2.9)$ & $62(5.7)$ & $45(8.7)$ & $18(9.8)$ & $18(19.4)$ \\
\hline Frequency of urination* & $33(6.8)$ & $90(8.3)$ & $73(14.1)$ & $34(18.5)$ & $19(20.4)$ \\
\hline Swelling in calves, legs, or feet* & $15(3.1)$ & $62(5.7)$ & $49(9.5)$ & $31(16.8)$ & $20(21.5)$ \\
\hline Coughing up sputum & $12(2.5)$ & $62(5.7)$ & $56(10.8)$ & $27(14.7)$ & $20(21.5)$ \\
\hline Pain or stiffness in neck ${ }^{*}$ & $32(6.6)$ & $128(11.8)$ & $72(13.9)$ & $49(26.6)$ & $22(23.7)$ \\
\hline Diarrhoea* & $24(5.0)$ & $99(9.1)$ & $70(13.5)$ & $31(16.8)$ & $22(23.7)$ \\
\hline Night sweats* & $22(4.5)$ & $88(8.1)$ & $65(12.5)$ & $25(13.6)$ & $22(23.7)$ \\
\hline Lightheadedness* & $11(2.3)$ & $62(5.7)$ & $53(10.2)$ & $37(20.1)$ & $22(23.7)$ \\
\hline Cough $^{*}$ & $36(7.4)$ & $98(9.0)$ & $83(16.0)$ & $41(22.3)$ & $23(24.7)$ \\
\hline Tingling or numbness in extremities* & $35(7.2)$ & $101(9.3)$ & $74(14.3)$ & $36(19.6)$ & $23(24.7)$ \\
\hline Leg cramps* & $31(6.4)$ & $103(9.5)$ & $55(10.6)$ & $31(16.8)$ & $23(24.7)$ \\
\hline Shortness of breath with exercise* & $11(2.3)$ & $59(5.4)$ & $68(13.1)$ & $37(20.1)$ & $23(24.7)$ \\
\hline Joint pain or swelling* & $42(8.7)$ & $140(12.9)$ & $81(15.6)$ & $45(24.5)$ & $25(26.9)$ \\
\hline Sleeping pattern changes* & $21(4.3)$ & $126(11.6)$ & $78(15.1)$ & $47(25.5)$ & $25(26.9)$ \\
\hline Tingling or pins and needles* & $30(6.2)$ & $91(8.4)$ & $75(14.5)$ & $36(19.6)$ & $25(26.9)$ \\
\hline Appetite changes* & $9(1.9)$ & $36(3.3)$ & $35(6.8)$ & $25(13.6)$ & $26(28.0)$ \\
\hline Heat or cold intolerance* & $17(3.5)$ & $85(7.8)$ & $65(12.5)$ & $36(19.6)$ & $27(29.0)$ \\
\hline Shortness of breath* & $8(1.7)$ & $51(4.7)$ & $57(11.0)$ & 34 (18.5) & $27(29.0)$ \\
\hline Bloating* & $26(5.4)$ & $104(9.6)$ & $61(11.8)$ & $41(22.3)$ & $29(31.2)$ \\
\hline Body image concerns* & $9(1.9)$ & $40(3.7)$ & $46(8.9)$ & $33(17.9)$ & 29 (31.2) \\
\hline Nasal stuffiness ${ }^{*}$ & $70(14.5)$ & $218(20.1)$ & $141(27.2)$ & $53(28.8)$ & $30(32.3)$ \\
\hline Urination at night* & $69(14.3)$ & $172(15.8)$ & $105(20.3)$ & $43(23.4)$ & $30(32.3)$ \\
\hline Muscle or joint pain* & $71(14.7)$ & $225(20.7)$ & $142(27.4)$ & $61(33.2)$ & $31(33.3)$ \\
\hline Memory change* & $9(1.9)$ & $52(4.8)$ & $55(10.6)$ & $41(22.3)$ & $32(34.4)$ \\
\hline
\end{tabular}


Table 2 Continued

\begin{tabular}{|c|c|c|c|c|c|}
\hline & $\begin{array}{l}\text { PHQ-9 } 0 \\
(\mathrm{~N}=484)\end{array}$ & $\begin{array}{l}\text { PHQ-9 1-4 } \\
(\mathrm{N}=1086)\end{array}$ & $\begin{array}{l}\text { PHQ-9 5-9 } \\
(\mathrm{N}=518)\end{array}$ & $\begin{array}{l}\text { PHQ-9 10-14 } \\
(\mathrm{N}=184)\end{array}$ & $\begin{array}{l}\text { PHQ-9 15+ } \\
(\mathrm{N}=93)\end{array}$ \\
\hline Headache* & $28(5.8)$ & $151(13.9)$ & $104(20.1)$ & $44(23.9)$ & $38(40.9)$ \\
\hline Fatigue $^{*}$ & $10(2.1)$ & $129(11.9)$ & $113(21.8)$ & $65(35.3)$ & $38(40.9)$ \\
\hline Stiffness* & $76(15.7)$ & $267(24.6)$ & $121(23.4)$ & $63(34.2)$ & 39 (41.9) \\
\hline Backache* & $43(8.9)$ & $183(16.9)$ & $148(28.6)$ & $63(34.2)$ & $39(41.9)$ \\
\hline Nervousness* & $4(0.8)$ & $46(4.2)$ & $51(9.8)$ & $39(21.2)$ & $43(46.2)$ \\
\hline Mood change* & $6(1.2)$ & $36(3.3)$ & $60(11.6)$ & $42(22.8)$ & $44(47.3)$ \\
\hline Difficulty concentrating ${ }^{*}$ & $8(1.7)$ & $58(5.3)$ & 74 (14.3) & $54(29.3)$ & $50(53.8)$ \\
\hline Neck or low back pain* & $73(15.1)$ & $264(24.3)$ & $185(35.7)$ & $73(39.7)$ & $51(54.8)$ \\
\hline Tension $^{\star}$ & $10(2.1)$ & $84(7.7)$ & $86(16.6)$ & $44(23.9)$ & $51(54.8)$ \\
\hline Lack of energy* & $6(1.2)$ & $92(8.5)$ & $115(22.2)$ & $74(40.2)$ & $53(57.0)$ \\
\hline
\end{tabular}

Data presented as no (\%).

$P$ values for trend were calculated with the use of Spearman correlation or Cochrane-Armitage tests, where appropriate.

${ }^{*} \mathrm{P}$ value for trend $<0.0001$.

ADHD, attention deficit hyperactivity disorder; COPD, chronic obstructive pulmonary disease; GERD, gastro-oesophageal reflux disease;

PHQ-9, Patient Health Questionnaire-9; PTSD, post-traumatic stress disorder.

offers an opportunity to better understand how different aspects of distress track similarly or differently over time.

An important aspect of our examination is how the binary division of PHQ-9 into labels of depression or 'not depression' leaves significant content unattended. While the division of PHQ-9 scores at 10 provides good discrimination with regard to a clinical diagnosis of Major Depressive Disorder, ${ }^{6}{ }^{10}$ the gradient between a score of 0 and 10 contains relevant information about distress levels. Such a finding is not novel, ${ }^{20}$ but a reminder that singular focus on a binary classification tends to obscure important information. For example, demographic data demonstrate how females and younger participants had higher PHQ-9 scores, yet whether this finding represents a different approach to revealing concerns or more significant distress is unclear.

\begin{tabular}{|c|c|c|c|c|c|}
\hline & PHQ-9 0 (N=484) & PHQ-9 1-4 (N=1086) & PHQ-9 5-9 (N=518) & PHQ-9 10-14 (N=184) & PHQ-9 15+ (N=93) \\
\hline 6 min walk* & $485.5(444.0,543.2)$ & $480.0(431.7,530.3)$ & $\begin{array}{l}465.0(422.0 \\
517.6)\end{array}$ & $460.1(403.8,511.9)$ & $\begin{array}{l}443.0(391.2, \\
492.4)\end{array}$ \\
\hline 30 s chair stand* & $15.0(12.0,18.0)$ & $14.0(12.0,17.0)$ & $13.0(11.0,16.0)$ & $13.0(10.0,16.0)$ & $12.0(10.0,15.0)$ \\
\hline $\begin{array}{l}\text { Mean leg balance } \\
\text { time } †\end{array}$ & $60.0(23.5,60.0)$ & $55.0(21.4,60.0)$ & $50.5(14.0,60.0)$ & $49.5(11.0,60.0)$ & $44.0(12.5,60.0)$ \\
\hline Handgrip† & $35.8(27.8,44.4)$ & $32.5(26.3,42.5)$ & $32.3(26.4,42.8)$ & $33.4(25.6,40.7)$ & $30.0(22.0,39.7)$ \\
\hline Sit-rise score & $8.0(6.0,9.0)$ & $8.0(6.0,9.0)$ & $8.0(6.0,9.0)$ & $8.0(6.0,9.0)$ & $7.5(5.0,9.0)$ \\
\hline EF at rest, \% & $59.0(55.8,60.5)$ & $58.8(55.9,60.5)$ & $59.2(56.1,60.5)$ & $59.2(56.0,61.4)$ & $58.9(56.0,60.9)$ \\
\hline $\begin{array}{l}\text { Mean steps in first } \\
30 \text { days }^{*}\end{array}$ & $\begin{array}{l}8398.0(6560.6 \\
10709.6)\end{array}$ & $\begin{array}{l}8172.2(6220.6 \\
10335.7)\end{array}$ & $\begin{array}{l}7666.7 \text { (5128.2, } \\
9862.5)\end{array}$ & $\begin{array}{l}7008.4 \text { (4673.5, } \\
9821.8)\end{array}$ & $\begin{array}{l}6498.1 \text { (4684.1, } \\
9044.5)\end{array}$ \\
\hline FEV1/FVC & $0.8(0.7,0.8)$ & $0.8(0.7,0.8)$ & $0.8(0.7,0.8)$ & $0.8(0.7,0.8)$ & $0.8(0.7,0.8)$ \\
\hline $\mathrm{ABI} \dagger$ & $1.1(1.1,1.2)$ & $1.1(1.1,1.2)$ & $1.1(1.1,1.2)$ & $1.1(1.0,1.2)$ & $1.1(1.0,1.2)$ \\
\hline
\end{tabular}

Data presented as median (25th, 75th percentile).

$P$ values for trend were calculated with the use of Spearman correlation or Cochrane-Armitage tests, where appropriate.

${ }^{*} \mathrm{P}$ value for trend $<0.01$

$\dagger P$ value for trend $<0.001$.

$\mathrm{ABI}$, ankle brachial index; EF, ejection fraction; FEV1, forced expiratory volume in 1s; FVC, forced vital capacity; PHQ-9, Patient Health Questionnaire. 


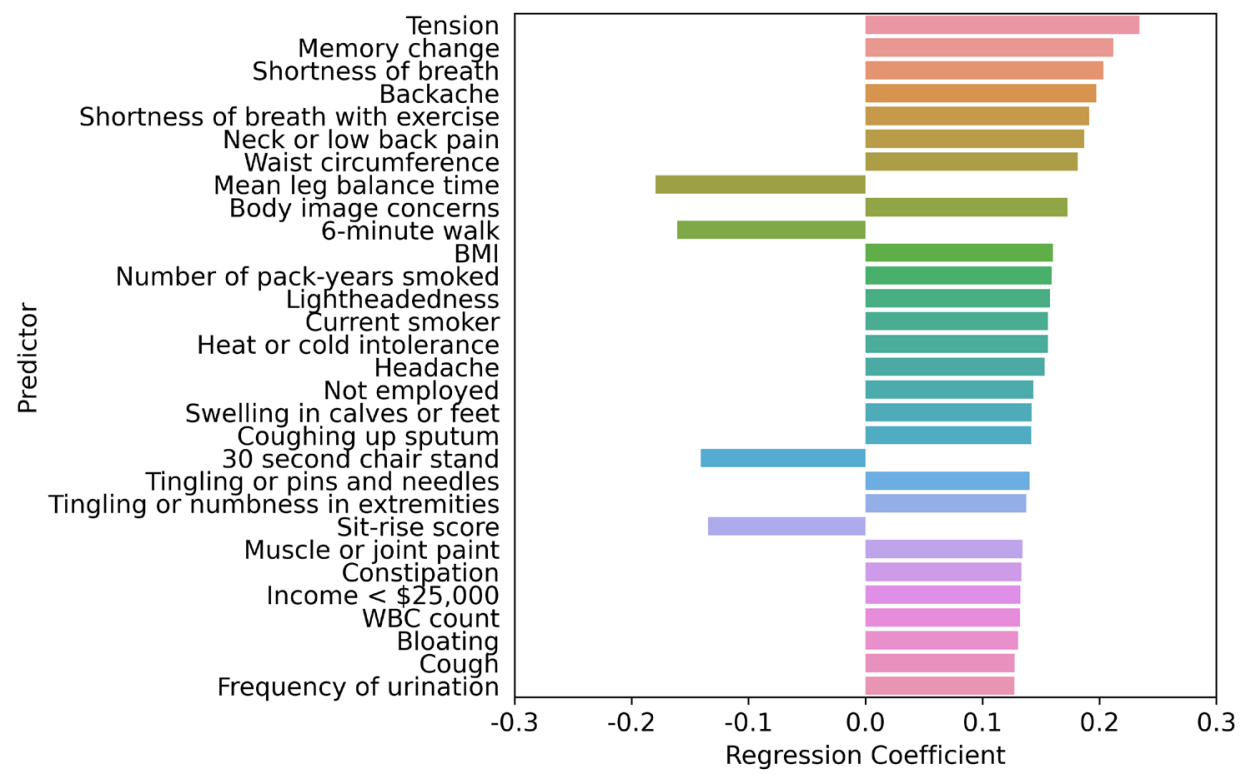

Figure 1 Top 30 factors associated with PHQ-9 score adjusted by age, sex, and age by sex interaction. LASSO regression model one comprised age, sex, and age by sex interaction. The LASSO-predicted value was used to estimate a covariateadjusted effect for all other candidate variables. BMI, body mass index; LASSO, least absolute shrinkage and selection operator; PHQ-9, Patient Health Questionnaire-9; WBC, white blood cell.

The higher scores associated with elevated heart rates, body mass index and abdominal girth were expected and consistent with previous literature. In general, PHQ-9 scores aligned with musculoskeletal or central and peripheral nervous system disorders, ${ }^{21}{ }^{22}$ but less with cardiovascular disease, cancer and other more organ-focused conditions. The previously widely reported association with obesity and diabetes was confirmed in our study. ${ }^{23-26}$ We found low PHQ-9 scores in a small, but noticeable proportion of the population with a previous diagnosis of depression, which could represent diagnostic errors, inaccurate reporting of medical history or recovery from a previous episode of depression.

The profound relationship between PHQ-9 score and a host of common symptoms ${ }^{27-31}$ is also expected, but the continuous nature of the relationship with PHQ-9 score and the depth of the relationships raise many issues that need further exploration. Significant predictors of

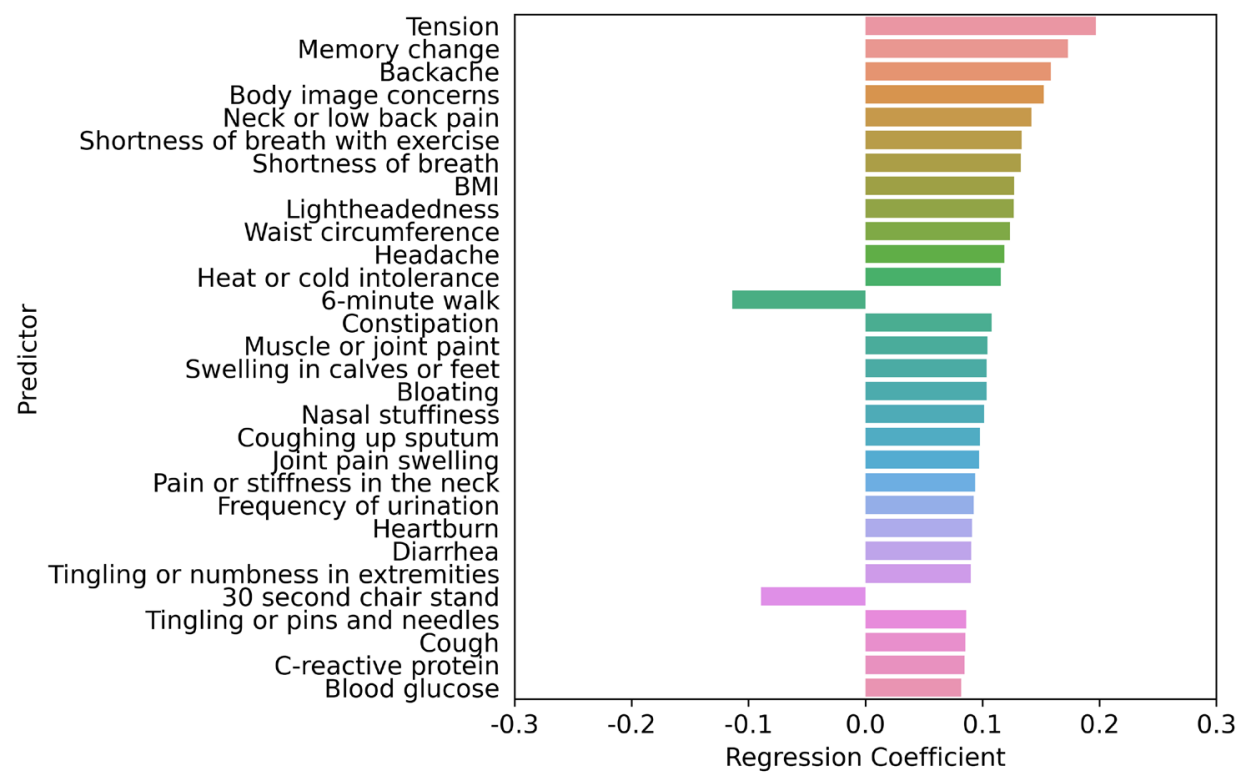

Figure 2 Top 30 factors associated with PHQ-9 score adjusted by age, sex, age by sex interaction, race, ethnicity, socioeconomic status and health behaviours. LASSO regression model two comprised age, sex, age by sex interaction, race, ethnicity, socioeconomic status and health behaviours. The LASSO-predicted value was used to estimate a covariate-adjusted effect for all other candidate variables. BMI, body mass index; LASSO, least absolute shrinkage and selection operator; PHQ-9, Patient Health Questionnaire-9. 


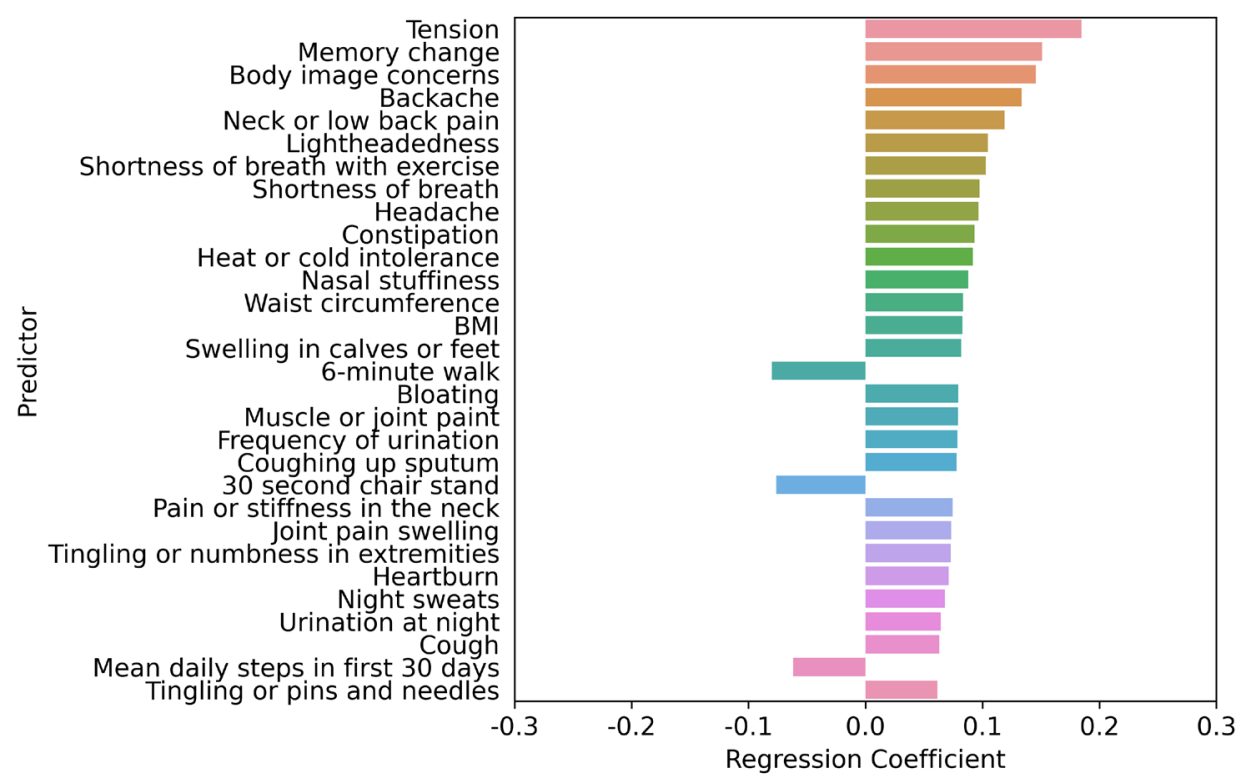

Figure 3 Top 30 factors associated with PHQ-9 score adjusted by age, sex, age by sex interaction, race, ethnicity, socioeconomic status, health behaviours, and medical conditions. LASSO regression model 3 comprised age, sex, age by sex interaction, race, ethnicity, socioeconomic status, health behaviours and medical conditions (except mental health disorder diagnoses or disorders directly related to mental health or depression). The LASSO-predicted value was used to estimate a covariate-adjusted effect for all other candidate variables. BMI, body mass index; LASSO, least absolute shrinkage and selection operator; PHQ-9, Patient Health Questionnaire-9.

PHQ-9 scores include a wide range of concerns encompassing neurological disease, musculoskeletal disease ${ }^{32} 33$ and psychological distress. A particularly notable finding is the progressive and highly significant relationship between reported memory loss and PHQ-9 score. Among people with PHQ-9 scores of 0 , only $1.9 \%$ reported memory change, while among those with PHQ-9 scores $>15$, memory change was reported in $34 \%$.

Finally, participants with higher PHQ-9 scores had impaired physical functioning, as indicated by a host

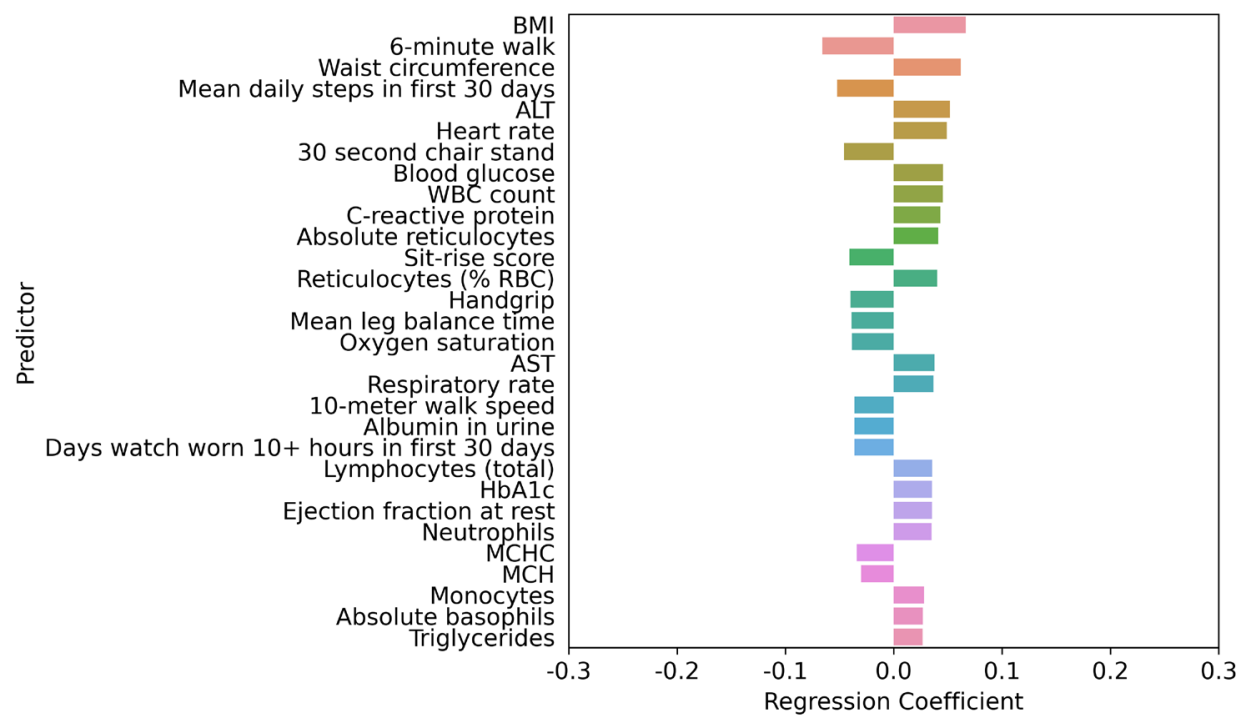

Figure 4 Top 30 factors associated with PHQ-9 score adjusted by age, sex, age by sex interaction, race, ethnicity, socioeconomic status, health behaviours, medical conditions, symptoms and allergies. LASSO regression model 4 comprised age, sex, age by sex interaction, race, ethnicity, socioeconomic status, health behaviours, medical conditions (except mental health disorder diagnoses or disorders directly related to mental health or depression), symptoms (except those that are directly related to mental health or depression) and allergies. The LASSO-predicted value was used to estimate a covariate-adjusted effect for all other candidate variables. ALT, alanine aminotransferase; AST, aspartate aminotransferase; BMI, body mass index; $\mathrm{HbA1c}$, haemoglobin A1c; LASSO, least absolute shrinkage and selection operator; $\mathrm{MCH}$, mean corpuscular haemoglobin; MCHC, mean corpuscular haemoglobin concentration; PHQ-9, Patient Health Questionnaire-9; RBC, red blood cell; WBC, white blood cell. 


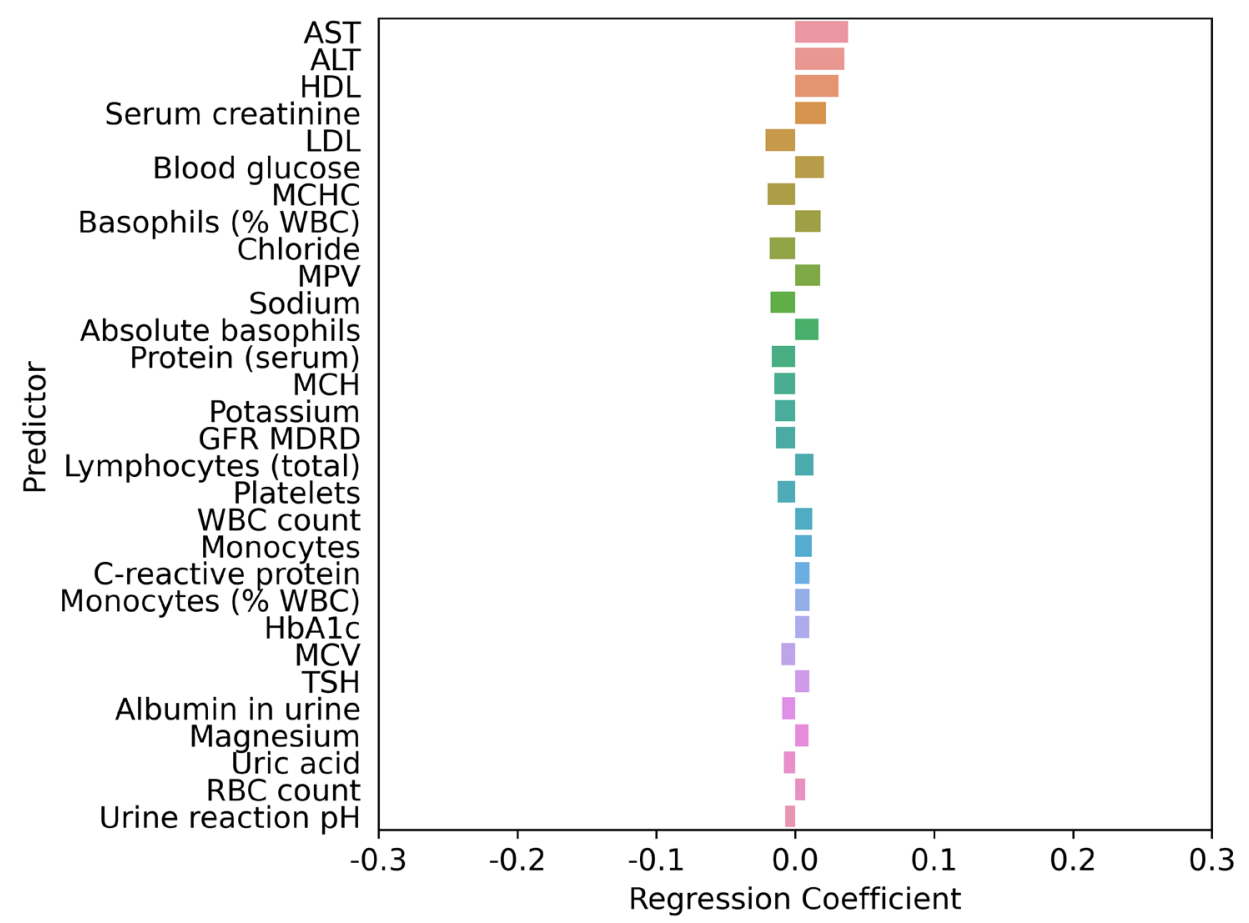

Figure 5 Top 30 factors associated with PHQ-9 score adjusted by age, sex, age by sex interaction, race, ethnicity, socioeconomic status, health behaviours, medical conditions, symptoms, allergies and physical function. LASSO regression model 5 comprised age, sex, age by sex interaction, race, ethnicity, socioeconomic status, health behaviours, medical conditions (except mental health disorder diagnoses or disorders directly related to mental health or depression), symptoms (except those that are directly related to mental health or depression), allergies, and physical function. The LASSO-predicted value was used to estimate a covariate-adjusted effect for all other candidate variables. ALT, alanine aminotransferase; AST, aspartate aminotransferase; GFR, glomerular filtration rate; HbA1c, haemoglobin A1c; HDL, high density lipoprotein; LASSO, least absolute shrinkage and selection operator; LDL, low density lipoprotein; $\mathrm{MCH}$, mean corpuscular haemoglobin; MCHC, mean corpuscular haemoglobin concentration; MCV, mean corpuscular volume; MDRD, modification of diet in renal disease; MPV, mean platelet volume; pH, potential hydrogen; PHQ-9, Patient Health Questionnaire-9; RBC, red blood cell; TSH, thyroidstimulating hormone; WBC, white blood cell.

of measurements, including daily steps, $6 \mathrm{~min}$ walk distance, ability to balance on one leg or propensity to exert a strong grip. Multiple previous studies have shown that depression and physical functioning are inter-related ${ }^{34-39}$ and that interventions to increase physical activity can improve depression status. ${ }^{40} 41$ Nevertheless, the overall picture of this study highlights the need for multimodal intervention to enable people with social disadvantages and physical comorbidities to improve physical and psychological function.

\section{Limitations}

This study has some limitations. First, the cross-sectional nature of the study limits our ability to assess the time course of these findings; however, follow-up is currently accruing. The time course of chronic disease and symptom progression in relation to the PHQ-9 will be of interest. Second, people with significant depression are probably less likely to volunteer, thereby limiting the breadth of depression observed in this study; BHS participants are volunteers from selected sites who express willingness to share data. Third, the population is generally representative of adult age, sex, race and ethnicity, but it is not a fully representative sample of the population; the differences between those who volunteer for digital technology studies and the general population are well known. ${ }^{42}$ Fourth, we acknowledge the high comorbidity of depression and anxiety, which is a potential bias; psychological comorbidities are important contextual factors to consider when assessing depression. We also lack detailed information on depression treatment, which is a potentially modifying factor. Finally, even though the PHQ-9 is a validated screening instrument, our study did not include psychiatric interviews.

\section{Conclusions}

PHQ-9 scores are related to multiple demographic, vital sign, and clinical measures that indicate poor physical status. BHS data provide a comprehensive picture of numerous interactive factors influencing PHQ-9 scores, thereby demonstrating how focusing on one chief complaint in hopes of improving depression status is likely futile, given that many common symptoms and physical limitations are profoundly integrated with depression status. The close association with symptoms often considered somatic raises a practical issue for clinical practice, and this is evident across the entire 
spectrum of PHQ-9 scores. When a high PHQ-9 score or other indicator of depression brings someone to the attention of a clinician, contextual awareness is critically important to provide an effective clinical intervention. When someone has significant neurological or musculoskeletal symptoms, assessment for depression should be a routine consideration. The complex associations across biological, clinical, behavioural and social factors stress the need for holistic evaluation of depression for individuals, as well as patient populations.

Collaborators Baseline Health Study Team: American Society of Clinical Oncology, Alexandria, VA, USA: Richard L. Schilsky. Duke University, School of Medicine, Durham, NC, USA: Jennifer Allen, MaryAnn Anderson, Kevin Anstrom, Lucus Araujo, Kristine Arges, Kaveh Ardalan, Bridget Baldwin, Suresh Balu, Mustafa R. Bashir, Manju Bhapkar, Robert Bigelow, Tanya Black, Rosalia Blanco, Gerald Bloomfield, Durga Borkar, Leah Bouk, Ebony Boulware, Nikki Brugnoni, Erin Campbell, Paul Campbell, Larry Carin, Tammy Jo Cassella, Tina Cates, Ranee Chatterjee Montgomery, Victoria Christian, John Choong, Michael Cohen-Wolkowiez, Elizabeth Cook, Scott Cousins, Ashley Crawford, Nisha Datta, Melissa Daubert, James Davis, Jillian Dirkes, Isabelle Doan, Marie Dockery, P. Murali Doraiswamy, Pamela S. Douglas, Shelly Duckworth, Ashley Dunham, Gary Dunn, Ryan Ebersohl, Julie Eckstrand, Vivienne Fang, April Flora, Emily Ford, Lucia Foster, Elizabeth Fraulo, John French, Geoffrey S. Ginsburg, Cindy Green, Latoya Greene, Jeffrey Guptill, Donna Hamel, Jennifer Hamill, Chris Harrington, Rob Harrison, Lauren Hedges, Brooke Heidenfelder, Adrian F. Hernandez, Cindy Heydary, Tim Hicks, Lina Hight, Deborah Hopkins, Erich S. Huang, Grace Huh, Jillian Hurst, Kelly Inman, Gemini Janas, Glenn Jaffee, Janace Johnson, Tiffanie Keaton, Michel Khouri, Daniel King, Jennifer Korzekwinski, Lynne H. Koweek, Anthony Kuo, Lydia Kwee, Dawn Landis, Rachele Lipsky, Desiree Lopez, Carolyn Lowry, Kelly Marcom, Keith Marsolo, Paige McAdams, Shannon McCall, Robert McGarrah, John McGugan, Dani Mee, Sabrena Mervin-Blake, Prithu Mettu, Mathias Meyer, Justin Meyers, Calire N. Miller, Rebecca Moen, Lawrence H. Muhlbaier, Michael Murphy, Ben Neely, L. Kristin Newby, Jayne Nicoldson, Hoang Nguyen, Maggie Nguyen, Lori O'Brien, Sumru Onal, Jeremey O'Quinn, David Page, Neha J. Pagidipati, Kishan Parikh, Sarah R. Palmer, Bray Patrick-Lake, Brenda Pattison, Michael Pencina, Eric D. Peterson, Jon Piccini, Terry Poole, Tom Povsic, Alicia Provencher, Dawn Rabineau, Annette Rich, Susan Rimmer, Fides Schwartz, Angela Serafin, Nishant Shah, Svati Shah, Kelly Shields, Steven Shipes, Peter Shrader, Jon Stiber, Lynn Sutton, Geeta Swamy, Betsy Thomas, Sandra Torres, Debara Tucci, Anthony Twisdale, Brooke Walker, Susan A. Whitney, Robin Williamson, Lauren Wilverding, Charlene A. Wong, Lisa Wruck. Ellen Young Gemini Group, USA: Jane Perlmutter. Health Collaboratory and Cancer 101, New York, NY, USA: Sarah Krug. Rare Dots, Inc., USA: S. Whitney Bowman-Zatzkin. Society of Participatory Medicine, USA: Sarah Krug. Stanford University, School of Medicine, Stanford, CA, USA: Themistocles Assimes, Vikram Bajaj, Maxwell Cheong, Millie Das, Manisha Desai, Alice C. Fan, Dominik Fleischmann, Sanjiv S. Gambhir, Garry Gold, Francois Haddad, David Hong, Curtis Langlotz, Yaping J. Liao, Rong Lu, Kenneth W. Mahaffey, David Maron, Rebecca McCue, Rajan Munshi, Fatima Rodriguez, Sumana Shashidhar, George Sledge, Susie Spielman, Ryan Spitler, Sue Swope, Donna Williams. University of Florida, College of Medicine, Gainesville, FL, USA: Carl J Pepine. University of Missouri, Children's Mercy Hospital, Kansas City, MO, USA: John D Lantos. University of Texas, Dell Medical School, Austin, TX, USA: Michael Pignone. University of Washington, Department of Biostatistics, Seattle, WA, USA: Patrick Heagerty. Vanderbilt University, School of Medicine, Nashville, TN, USA: Laura Beskow, Gordon Bernard. Verily Inc., South San Francisco, CA, USA: Kelley Abad, Giulia Angi, Robert M. Califf, Lawrence Deang, Joy Huynh, Manway Liu, Cherry Mao, Michael Magdaleno, William J. Marks, Jr., Jessica Mega, David Miller, Nicole Ong, Darshita Patel, Vanessa Ridaura, Scarlet Shore, Sarah Short, Michelle Tran, Veronica Vu, Celeste Wong. Harvard University, School of Medicine, Boston, MA, USA: Robert C. Green. Google Inc., Mountain View, CA, USA: John Hernandez. California Health and Longevity Institute, Westlake Village, CA, USA: Jolene Benge, Gislia Negrete, Gelsey Sierra, Terry Schaack.

Contributors RMC had full access to all of the data in the study and takes responsibility for the integrity of the data and the accuracy of the data analysis. RMC contributed to the conception and design of the study, the data analysis, the data interpretation, the manuscript drafting, and the critical revision of the manuscript. CW contributed to the conception and design of the study, data interpretation, the manuscript drafting, and the critical revision of the manuscript. MD contributed to the data interpretation, the manuscript drafting, and the critical revision of the manuscript. DSH contributed to the data interpretation, the manuscript drafting, and the critical revision of the manuscript. DPM contributed to the conception and design of the study, data interpretation, the manuscript drafting, and the critical revision of the manuscript. JLM contributed to the conception and design of the study, the supervision, data acquisition, data interpretation, the manuscript drafting and the critical revision of the manuscript. Guarantor is Robert M. Califf (RMC)

Funding This analysis was funded by Verily Life Sciences (South San Francisco, CA) in partnership with Stanford University and Duke University.

Competing interests RMC: Employee of Verily Life Sciences and Google Health; Board member for Cytokinetics, Centessa, Keystone Symposia, the Center for Policy Analysis on Trade and Health, Clinical Research Forum, One Fifteen, Launch and Scale Speedometer, Think Tank, Human Health and Potential, United Medicines, Medicxi, and Clinetic. CW: Employee of Verily Life Sciences. MD: Consulting fees from Verily Life Sciences, Neuronix, Apollo Health, VitaKey, Neuroglee, Transposon, Otsuka; Research grants from Avanir, Lilly, Avid, Salix; Holds stock in Evidation Health, Advera Health Analytics, Transposon Therapeutics, Marvel Biome; Board membership in Apollo; Coinventor on patents for diagnosis or treatment of neuropsychiatric disorders. DSH: Research funding from NIMH; Consulting for Little Otter. DPM: Employee of and holds stock in Verily Life Sciences. JLM: Employee of Verily Life Sciences.

Patient consent for publication Not applicable.

Ethics approval This study approved by wcg IRBWork order \#: 1-1379515-1IRB tracking \#: 20170163. Approval expires: 27 February 2022.

Provenance and peer review Not commissioned; externally peer reviewed.

Data availability statement Data are available on reasonable request. The Baseline Study data will be available to qualified researchers for exploratory analysis after the data are adequately curated and initial planned primary manuscripts are written. Qualified external researchers will be able to apply through applications reviewed by the Proposal Review and Publications Committee and Scientific Executive Committee.

Supplemental material This content has been supplied by the author(s). It has not been vetted by BMJ Publishing Group Limited (BMJ) and may not have been peer-reviewed. Any opinions or recommendations discussed are solely those of the author(s) and are not endorsed by BMJ. BMJ disclaims all liability and responsibility arising from any reliance placed on the content. Where the content includes any translated material, BMJ does not warrant the accuracy and reliability of the translations (including but not limited to local regulations, clinical guidelines, terminology, drug names and drug dosages), and is not responsible for any error and/or omissions arising from translation and adaptation or otherwise.

Open access This is an open access article distributed in accordance with the Creative Commons Attribution Non Commercial (CC BY-NC 4.0) license, which permits others to distribute, remix, adapt, build upon this work non-commercially, and license their derivative works on different terms, provided the original work is properly cited, appropriate credit is given, any changes made indicated, and the use is non-commercial. See: http://creativecommons.org/licenses/by-nc/4.0/.

ORCID iD

Robert M Califf http://orcid.org/0000-0003-0231-3724

\section{REFERENCES}

1 GBD 2017 Disease and Injury Incidence and Prevalence Collaborators. Global, regional, and national incidence, prevalence, and years lived with disability for 354 diseases and injuries for 195 countries and territories, 1990-2017: a systematic analysis for the global burden of disease study 2017. Lancet 2018;392:1789-858.

2 Perry PJ. The interaction of major depression and medical illness, 2005. Available: https://www.medscape.org/viewarticle/517033

3 Kampling $\mathrm{H}$, Baumeister $\mathrm{H}$, Bengel J, et al. Prevention of depression in adults with long-term physical conditions. Cochrane Database Syst Rev 2021;3:CD011246.

4 Zhdanava M, Kuvadia H, Joshi K, et al. Economic burden of treatment-resistant depression in privately insured U.S. patients with physical conditions. J Manag Care Spec Pharm 2020;26:996-1007.

5 Rosenblat JD, Kurdyak P, Cosci F, et al. Depression in the medically ill. Aust N Z J Psychiatry 2020;54:346-66.

6 Kroenke K, Spitzer RL, Williams JB. The PHQ-9: validity of a brief depression severity measure. J Gen Intern Med 2001;16:606-13. 
7 Huang FY, Chung $\mathrm{H}$, Kroenke $\mathrm{K}$, et al. Using the patient health questionnaire- 9 to measure depression among racially and ethnically diverse primary care patients. J Gen Intern Med 2006;21:547-52.

8 Patel JS, Oh Y, Rand KL, et al. Measurement invariance of the patient health questionnaire-9 (PHQ-9) depression screener in U.S. adults across sex, race/ethnicity, and education level: NHANES 2005-2016. Depress Anxiety 2019;36:813-23.

9 Levis B, Benedetti A. Thombs BD for the depression screening data (DEPRESSD) collaboration. accuracy of patient health questionnaire-9 (PHQ-9) for screening to detect major depression: individual participant data meta-analysis. BMJ 2019;365:1476.

10 Levis $\mathrm{B}$, Sun $\mathrm{Y}, \mathrm{He} \mathrm{C}$, et al. Accuracy of the PHQ-2 alone and in combination with the PHQ-9 for screening to detect major depression: systematic review and meta-analysis. JAMA 2020;323:2290-300

11 Arges K, Assimes T, Bajaj V, et al. The project baseline health study: a step towards a broader mission to map human health. NPJ Digit Med 2020;3:84.

12 Califf RM, Wong C, Doraiswamy PM, et al. Importance of social determinants in screening for depression. J Gen Intern Med 2021:1. doi:10.1007/s11606-021-06957-5

13 Armitage P. Tests for linear trends in proportions and frequencies. Biometrics 1955;11:375-86.

14 Liu L, Berger VW, Hershberger SL. Trend tests for counts and proportions. Wiley StatsRef: Statistics Reference Online, 2014.

15 Mukaka MM. Statistics corner: a guide to appropriate use of correlation coefficient in medical research. Malawi Med $\mathrm{J}$ 2012;24:69-71.

16 Brody DJ, Pratt LA, Hughes J. Prevalence of depression among adults aged 20 and over: United States, 2013-2016. NCHS data brief, no 303. Hyattsville, MD: National Center for Health Statistics, 2018.

17 Blanco C, Okuda M, Markowitz JC, et al. The epidemiology of chronic major depressive disorder and dysthymic disorder: results from the National epidemiologic survey on alcohol and related conditions. J Clin Psychiatry 2010;71:1645-56.

18 Spitzer RL, Kroenke K, Williams JB. Validation and utility of a selfreport version of PRIME-MD: the PHQ primary care study. primary care evaluation of mental disorders. patient health questionnaire. JAMA 1999;282:1737-44.

19 Siu AL, Bibbins-Domingo K, et al, US Preventive Services Task Force (USPSTF). Screening for depression in adults: US preventive services task force recommendation statement. JAMA 2016;315:380-7.

20 Thombs BD, Benedetti A, Kloda LA, et al. The diagnostic accuracy of the patient health Questionnaire-2 (PHQ-2), patient health Questionnaire-8 (PHQ-8), and patient health Questionnaire-9 (PHQ-9) for detecting major depression: protocol for a systematic review and individual patient data meta-analyses. Syst Rev 2014;3:124.

21 Hoshino E, Ohde S, Rahman M, et al. Variation in somatic symptoms by patient health questionnaire- 9 depression scores in a representative Japanese sample. BMC Public Health 2018;18:1406.

22 Leavens A, Patten SB, Hudson M, et al. Influence of somatic symptoms on patient health Questionnaire-9 depression scores among patients with systemic sclerosis compared to a healthy general population sample. Arthritis Care Res 2012;64:1195-201.

23 Snoek FJ, Bremmer MA, Hermanns N. Constructs of depression and distress in diabetes: time for an appraisal. Lancet Diabetes Endocrinol 2015;3:450-60.
24 Nouwen A, Winkley K, Twisk J, et al. Type 2 diabetes mellitus as a risk factor for the onset of depression: a systematic review and metaanalysis. Diabetologia 2010;53:2480-6.

25 Ciechanowski PS, Katon WJ, Russo JE. Depression and diabetes: impact of depressive symptoms on adherence, function, and costs. Arch Intern Med 2000;160:3278-85.

26 Lustman PJ, Anderson RJ, Freedland KE, et al. Depression and poor glycemic control: a meta-analytic review of the literature. Diabetes Care 2000;23:934-42.

27 Kroenke K, Spitzer RL, Williams JBW. The PHQ-15: validity of a new measure for evaluating the severity of somatic symptoms. Psychosom Med 2002;64:258-66.

28 Kocalevent R-D, Hinz A, Brähler E. Standardization of a screening instrument (PHQ-15) for somatization syndromes in the general population. BMC Psychiatry 2013;13:91.

29 Löwe B, Spitzer RL, Williams JBW, et al. Depression, anxiety and somatization in primary care: syndrome overlap and functional impairment. Gen Hosp Psychiatry 2008;30:191-9.

30 Ziebold C, Goldberg DP, Reed GM, et al. Dimensional analysis of depressive, anxious and somatic symptoms presented by primary care patients and their relationship with ICD-11 PHC proposed diagnoses. Psychol Med 2019;49:764-71.

31 Kroenke K, Spitzer RL, Williams JBW, et al. The patient health questionnaire somatic, anxiety, and depressive symptom scales: a systematic review. Gen Hosp Psychiatry 2010;32:345-59.

32 Hartvigsen J, Christensen K. Pain in the back and neck are with us until the end: a nationwide interview-based survey of Danish 100-year-olds. Spine 2008;33:909-13.

33 Reichborn-Kjennerud T, Stoltenberg C, Tambs K, et al. Back-neck pain and symptoms of anxiety and depression: a population-based twin study. Psychol Med 2002;32:1009-20.

34 Zhang J, Yen ST. Physical activity, gender difference, and depressive symptoms. Health Serv Res 2015;50:1550-73.

35 Farmer ME, Locke BZ, Mościcki EK, et al. Physical activity and depressive symptoms: the NHANES I epidemiologic follow-up study. Am J Epidemiol 1988;128:1340-51.

36 Helgadóttir B, Forsell Y, Ekblom Örjan. Physical activity patterns of people affected by depressive and anxiety disorders as measured by accelerometers: a cross-sectional study. PLoS One 2015;10:e0115894.

37 Sieverdes JC, Ray BM, Sui X, et al. Association between leisure time physical activity and depressive symptoms in men. Med Sci Sports Exerc 2012;44:260-5.

38 Sun G-Z, Ye N, Wu S-J, et al. 10-year ASCVD risk is positively correlated with depressive symptoms in a large general population. BMC Psychiatry 2019;19:125.

39 Jha MK, Qamar A, Vaduganathan M, et al. Screening and management of depression in patients with cardiovascular disease: JACC state-of-the-art review. J Am Coll Cardiol 2019;73:1827-45.

40 Wegner M, Helmich I, Machado S, et al. Effects of exercise on anxiety and depression disorders: review of meta- analyses and neurobiological mechanisms. CNS Neurol Disord Drug Targets 2014;13:1002-14.

41 Blumenthal JA, Babyak MA, Doraiswamy PM, et al. Exercise and pharmacotherapy in the treatment of major depressive disorder. Psychosom Med 2007;69:587-96.

42 Fareed N, Swoboda CM, Jonnalagadda P, et al. Persistent digital divide in health-related Internet use among cancer survivors: findings from the health information national trends survey, 2003-2018. $J$ Cancer Surviv 2021;15:87-98. 